\title{
A Study of Awareness and Practice of Infection Control of Dental Hygiene Students during Clinical Practice
}

\author{
Min-Seo Kim \\ Department of Oral Health, Dankook University, Cheonan, Korea
}

Objective: The purpose of this study is to minimize dental infection in hospitals and develop infection control curriculum programs.

\begin{abstract}
Methods: By investigating the perception and practice of dental infection management in clinical practice sites for dental hygiene students who have experienced clinical practice. It was implemented to provide basic data. A survey was conducted on dental hygiene students who actually experienced clinical practice at a three-year university in Gyeongbuk, and students who practiced in dentistry, dental hospitals, university hospitals, or general hospitals were classified to recognize the management of dental infections in each practice area. And the degree of practice were compared.
\end{abstract}

Results: As a result of the study, students had a lot of experience in infection prevention education, but education was conducted in clinical practice sites rather than in schools, and the degree of practice of infection prevention management was higher in university hospitals or dental hospitals, and there were differences according to the practice sites.

Conclusion: Therefore, in order for students to increase their awareness and practice of hepatitis management in clinical practice areas, infection control guidelines should be prepared in schools, distributed to clinical institutions, and managed through training personnel. Infection control education in connection with clinical practice in schools It is believed that education should be conducted by systematically preparing courses.

Keywords: dental hygiene, clinical practice, dental infection management, infection management curriculum

\section{Introduction}

There is always a risk of cross-infection due to internal contamination, and the risk of cross-infection of infectious diseases such as hepatitis B and HIV is high, so dental workers

\section{Corresponding author Min-Seo Kim}

E-mail: kms790204@naver.com

(iD) https://orcid.org/0000-0003-4206-3731

Received June 11, 2021, Revised June 21, 2021, Accepted June 28, 2021 can be another vector of infection [1]. In addition, dental treatment has a high risk of infection due to environmentally close proximity of practitioners, assistants, and dental consumers [2]. Various pathogenic microorganisms present in blood or saliva during the treatment process spread into the air in the form of aerosols and dusts. It pollutes my environment, the instruments used are sharp, and most of the procedure is bleeding and is exposed to a relatively high risk of infection [3]. Therefore, since the 1970s, the United States has established an academic and institutional foundation through hospital infection control research and practice. Prevention guidelines were created and operated [4], Currently, practical guidelines

Copyright (C) 2021. Korean Academy of Preventive Dentistry. All rights reserved.

This is an Open Access article distributed under the terms of the Creative Commons Attribution Non-Commercial License (http://creativecommons.org/licenses/ by-nc/4.0) which permits unrestricted non-commercial use, distribution, and reproduction in any medium, provided the original work is properly cited. 
and procedures for the protection of dental workers are adapted to the dental medical environment and even inspections are carried out [5]. Therefore, in July 2006, Korea also established standards for prevention of infection for the disinfection and sterilization of dental equipment and equipment used in dental clinics, and began to establish standards for preventing infection in dental clinics to improve the hygiene level of dental clinics and to prevent infection [6,7]. After various implementation procedures, the Dental Infection Management Manual was finally created in June 2020. However, in the dental medical field, as the invasive manipulation and use of medical devices have increased due to the development of medical technology, and the increasing number of test technologies dealing with blood, the proportion of infections caused by blood has increased. As the number of hospital-related workers increases, the rate of hospital infection is increasing [8]. In order to improve the quality of medical care in this way, continuous improvement measures are being made through the dental medical institution certification system, and a study on the development of quality improvement management indicators for dental medical institutions utilizing some dental infection management parts [9]. To improve, the need to develop a dental hygienist infection control practice scale was emphasized. And, looking at the domestic research trends on the prevention of infection in the dental clinic, most of the researches targeting dental hygienists and nurses [10-16] include the American Dental Association or American Disease. This is a survey of awareness and actual conditions based on the standards of the management center or the standards set by researchers. However, in Korea, there is a very lack of comprehensive research including infection control conditions during clinical practice of dental hygiene and students. In particular, despite the preclinical education for infection prevention in the same environment as the dental clinic at the university, the current university education environment is limited to be applied to the clinic. In addition, even though a standard for dental infection prevention has been established and the risk of infection is recognized, if the will to implement it is insufficient due to various environmental factors, infection control in the dental clinic is a difficult problem to solve. Infection prevention is not achieved by investing a large amount of money, but only by people who can properly use and practice infection knowledge, skills, endurance efforts, and constant interest in hospital hygiene [17]. In practice, dental hygienists in dental clinical practice are performing infection control tasks such as disinfection, sterilization, cleaning, instrument management, and extract management, and are performing as infection managers during the clinical practice period of students.
Therefore, this study investigates the perception and practice of dental infection prevention of dental hygiene students who have experienced clinical practice to reduce the incidence of infection in the dental clinic, and what are necessary in carrying out the guidelines necessary for infection prevention during clinical practice. We will identify the problem and use it as basic data necessary for education before correct clinical practice.

\section{Materials and Methods}

\section{Research subject and data collection}

This study was aimed at students of the 2.3th grade dental hygiene who actually experienced clinical practice at a threeyear university located in Gyeongbuk, and the students performed practice at different dental medical institutions. The number of study subjects was selected with 100 people. Data collection was conducted for 3 months from July 1 to September 30,2020 , of which 100 copies were collected and 98 copies were used, excluding 2 copies with insufficient questionnaire responses. As for the survey method, the researcher handed out a structured questionnaire to the subjects, explained the purpose and purpose of the study, asked for consent, and then collected it in a self-written form.

\section{Research method}

A structured questionnaire was used as a tool for the study, and the questionnaire was modified and supplemented with reference to Park [18]'s research. The composition of the questionnaire consists of 3 questions about the general characteristics of the study subjects, 4 questions about infection prevention awareness, 4 questions about infection control status in the clinical training site, 15 questions about infection prevention attitudes in the clinical training site, and the equipment used in hospitals. It consisted of 10 questions asking about the degree of practice on sterilization and disinfection, and the reliability of the research tool was Cronbach's $\alpha=.913$, and the measurement tool was reliable.

\section{Data analysis}

Statistical analysis of the collected data was performed using the statistical program SPSS (Statistical Packages for Social Science 19.0. SPSSINC. USA). To grasp the general characteristics of the study subjects, the frequency and percentage of understanding were calculated, and the frequency and percentage were calculated for the education and health check-ups for dental hygiene and student infection prevention, and the perception of dental infection prevention. 
The frequency and percentage were calculated for education on infection prevention, health check-ups, and perceptions on dental infection prevention. In addition, ANOVA and Sheffe were performed for the practice of infection prevention in each area and the disinfection and sterilization of instruments in the clinical practice site.

\section{Results}

\section{General characteristics of the research subject}

As for the general characteristics of the subjects, the gender was $95(96.9 \%)$, mostly female, and the practical semester was $66(67.3 \%)$ in the first semester of the second year, and 32 $(32.7 \%)$ in the second semester of the second year. Clinical practice sites were found in 34 dental clinics (34.7\%), 41 dental clinics (41.8\%), and 23 general hospitals and university hospitals $(23.5 \%)$ (Table 1$)$.

\section{Infection prevention education of clinical trainees and awareness of infection in hospital \\ 1) Matters concerning dental infection management education and medical examination of clinical trainees}

As for whether or not clinical trainees had received dental infection management education before going to clinical practice, 92 people (93.9\%) said yes, and 86 people (87.8\%) had the highest dental infection management education path in the dental practice. As for whether they had regular health checkups before going to clinical practice, 59 people $(60.2 \%)$ answered 'yes', and 39 people (39.8\%) answered 'No'. The most common reason for not receiving regular health checkups was "because they thought they were healthy" with 80 (81.6\%), and $16(16.3 \%)$ with "not likely to be infected" (Table 2 ).

\section{2) Clinicians' perception of hospital infection}

As a result of analyzing the infection control status of the clinical practice site, the diseases considered to be the most in-

Table 1. The dental infection management education

\begin{tabular}{llc}
\hline \multicolumn{1}{c}{ Variable } & \multicolumn{1}{c}{ Classification } & $\mathrm{n}(\%)$ \\
\hline Gender & Female & $95(96.9)$ \\
& Male & $3(3.1)$ \\
Grade & Two- 1 grade & $66(67.3)$ \\
& Two- 2 grade & $32(32.7)$ \\
Dental practice & Dental clinic & $34(34.7)$ \\
place & Dental hospital & $41(41.8)$ \\
& General hospital and university & $23(23.5)$ \\
& hospital & \\
& &
\end{tabular}

fectious in dentistry were 'HIV' 38 (38.8\%), 'Influenza' 31 (31.6\%), and 'Tuberculosis' 18 (18.4\%). The infection route of the clinical practice site was 'contaminated needles or sharp instruments' 42 people (42.9\%), and 'through skin contact through blood, saliva and mucus' 34 people (34.7\%). 38 (38.8\%) said that it was 'well-implemented' and 37 (37.8\%) said that it was 'very well implemented' (Table 3 ).

Table 2. Matters related to medical checkup and education to prevent infection of clinical trainees

\begin{tabular}{clc}
\hline \multicolumn{1}{c}{ Variable } & \multicolumn{1}{c}{ Classification } & $\mathrm{n}(\%)$ \\
\hline $\begin{array}{c}\text { Education to } \\
\text { dental infection }\end{array}$ & Yes & $92(93.9)$ \\
management & & $6(6.1)$ \\
Educational & School education & \\
pathway to & Dental clinic & $4(4.1)$ \\
dental infection & Extra education & $86(87.8)$ \\
management & Internet education & $3(3.1)$ \\
Regular health & Yes & $5(5.1)$ \\
checks & No & $59(60.2)$ \\
Why won't do a & Healthy thought & $39(39.8)$ \\
medical & Financial burden & $80(81.6)$ \\
check-up & Reluctance to get health checks & $1(1.0)$ \\
regularly & That would be unlikely to be infected & $16(1.0)$ \\
\hline
\end{tabular}

Table 3. The awareness of the infection in hospital of clinical practice

\begin{tabular}{llc}
\hline \multicolumn{1}{c}{ Variable } & \multicolumn{1}{c}{ Classification } & $\mathrm{n}(\%)$ \\
\hline $\begin{array}{ll}\text { Infectious disease that } \\
\text { thought it was the }\end{array}$ & HIV & $38(38.8)$ \\
highest in the dental & Influenza & $11(11.2)$ \\
clinic & Tuberculosis & $31(31.6)$ \\
Reason for hospital & Dirty needles or sharp & $18(18.4)$ \\
infections & instrument & $42(42.9)$ \\
& Blood or saliva, and & $34(34.7)$ \\
& contaminants are in contact & \\
& with skin or mucous & \\
& membranes & $22(22.4)$ \\
Sanitary control of & Polluted air mass & $37(37.8)$ \\
devices and equip & Done very well & Done well \\
ment in hospital & Normal & $22(22.4)$ \\
& Not well implemented & $1(1.0)$ \\
Total & & $98(100)$ \\
\hline
\end{tabular}


3. Matters concerning the status of infection prevention management in hospital according to clinical practice sites and sterilization and disinfection of instruments used for patients

1) Matters concerning the attitude of infection prevention management according to clinical practice

As a result of comparing matters on infection prevention attitudes according to clinical practice sites, university hospitals and general hospitals scored 3.96 points for unit chairs, 3.87 points for batting tables, and 3.91 points for protective equipment stained with saliva or blood. There was a statistically significant difference between the dental clinic and the dental clinic $(\mathrm{p}<0.03)$. In extract management, dental hospitals scored 3.88 points, and dental hospitals, university hospitals, and general hospitals were higher than dental clinics before and after treatment. In terms of handwashing, university hospitals and general hospitals scored "before treatment" 3.74 points and "after treatment" 3.83 points, which were higher than dental clinics and dental hospitals. University hospitals and general hospitals were higher than dental clinics and dental hospitals in both 'when preparing for setting', 'before treatment' and 'after treatment' in surface disinfection, and 'protective goggles', 'disposable towels', and 'hall In both 'Towels', university hospitals and general hospitals were higher than dental clinics and dental hospitals (Table 4).

Table 4. Matters concerning care to prevent infection in clinical practice

\begin{tabular}{|c|c|c|c|c|c|}
\hline Variable & Classification & Dental clinic & Dental hospital & $\begin{array}{l}\text { General hospital } \\
\text { and university } \\
\text { hospital }\end{array}$ & p-value \\
\hline \multirow[t]{3}{*}{ Cleaning } & Unit chair & $3.74 \pm 0.51$ & $3.88 \pm 0.40$ & $3.96 \pm 0.20$ & 0.11 \\
\hline & Spittoon & $3.59 \pm 0.55$ & $3.76 \pm 0.62$ & $3.87 \pm 0.34$ & 0.15 \\
\hline & Saliva or blood-stained protective equipment & $3.65 \pm 0.54^{\mathrm{a}}$ & $3.88 \pm 0.40^{\mathrm{b}}$ & $3.91 \pm 0.28^{\mathrm{C}}$ & $0.03 *$ \\
\hline \multirow[t]{3}{*}{ Hospital supplies care } & Infections waste and ordinary waste & $3.82 \pm 0.38$ & $3.88 \pm 0.40$ & $3.87 \pm 0.34$ & 0.81 \\
\hline & Before the treatment & $3.68 \pm 0.47$ & $3.78 \pm 0.47$ & $3.78 \pm 0.42$ & 0.56 \\
\hline & After the treatment & $3.65 \pm 0.64$ & $3.83 \pm 0.38$ & $3.83 \pm 0.38$ & 0.22 \\
\hline \multirow[t]{3}{*}{ Hand washing } & Set-up preparation (once a day) & $3.79 \pm 0.64$ & $3.80 \pm 0.40$ & $3.83 \pm 0.49$ & 0.97 \\
\hline & Before the treatment & $3.68 \pm 0.58$ & $3.66 \pm 0.61$ & $3.74 \pm 0.44$ & 0.86 \\
\hline & After the treatment & $3.65 \pm 0.59$ & $3.76 \pm 0.53$ & $3.83 \pm 0.38$ & 0.43 \\
\hline \multirow[t]{3}{*}{ Surface disinfection } & Set-up preparation (once a day) & $3.85 \pm 0.43$ & $3.88 \pm 0.33$ & $3.96 \pm 0.20$ & 0.53 \\
\hline & Before the treatment & $3.76 \pm 0.55$ & $3.80 \pm 0.55$ & $3.96 \pm 0.20$ & 0.34 \\
\hline & After the treatment & $3.82 \pm 0.45$ & $3.73 \pm 0.63$ & $3.91 \pm 0.28$ & 0.38 \\
\hline \multirow{3}{*}{$\begin{array}{l}\text { Wearing protective } \\
\text { equipment hygiene at treat }\end{array}$} & Protective goggles & $3.21 \pm 0.88$ & $3.46 \pm 0.63$ & $3.61 \pm 0.49$ & 0.09 \\
\hline & Disposable towel & $3.53 \pm 0.86$ & $3.54 \pm 0.71$ & $3.70 \pm 0.47$ & 0.64 \\
\hline & Hole towel & $3.47 \pm 0.89$ & $3.54 \pm 0.74$ & $3.57 \pm 0.50$ & 0.88 \\
\hline
\end{tabular}

Unit: score by ANOVA, ${ }^{*} \mathrm{p}<0.05 .{ }^{\mathrm{a}, \mathrm{b}, \mathrm{c}} \mathrm{The}$ same characters are not significant by Scheffe test. Cleaning: $\mathrm{a}<\mathrm{b}, \mathrm{c}$.

Table 5. Matters concerning sterilization and disinfection of the equipment used for patient in clinical practice

\begin{tabular}{|c|c|c|c|c|}
\hline Classification & Dental clinic & Dental hospital & $\begin{array}{c}\text { General hospital and } \\
\text { university hospital }\end{array}$ & p-value \\
\hline Ultrasonic scaler tip & $3.62 \pm 0.55$ & $3.85 \pm 0.35$ & $3.83 \pm 0.38$ & 0.05 \\
\hline Impression tray & $3.79 \pm 0.47$ & $3.80 \pm 0.55$ & $3.78 \pm 0.60$ & 0.98 \\
\hline Handle implement & $3.91 \pm 0.37$ & $3.88 \pm 0.51$ & $4.00 \pm 0.00$ & 0.50 \\
\hline Glass ball & $3.29 \pm 0.93$ & $3.54 \pm 0.84$ & $3.78 \pm 0.60$ & 0.09 \\
\hline Rubber cup & $3.47 \pm 0.89$ & $3.46 \pm 0.80$ & $3.91 \pm 0.41$ & 0.05 \\
\hline Coton roll & $3.53 \pm 0.70^{\mathrm{a}}$ & $3.88 \pm 0.33^{b}$ & $3.87 \pm 0.45^{c}$ & $0.00 *$ \\
\hline Guze & $3.65 \pm 0.54^{\mathrm{a}}$ & $3.88 \pm 0.33^{b}$ & $3.96 \pm 0.20^{c}$ & $0.00^{*}$ \\
\hline Bur & $3.82 \pm 0.38$ & $3.88 \pm 0.33$ & $4.00 \pm 0.00$ & 0.11 \\
\hline Suction tip & $3.91 \pm 0.28$ & $3.88 \pm 0.40$ & $3.96 \pm 0.20$ & 0.65 \\
\hline Handpiece & $3.62 \pm 0.60$ & $3.71 \pm 0.55$ & $3.83 \pm 0.38$ & 0.36 \\
\hline
\end{tabular}

Unit : score by ANOVA, ${ }^{*} \mathrm{p}<0.05 .{ }^{\mathrm{a}, \mathrm{b}, \mathrm{c}}$ The same characters are not significant by Scheffe test. Coton roll: $\mathrm{a}<\mathrm{c}, \mathrm{b}$. Guze: $\mathrm{a}<\mathrm{c}$. 
2) Matters concerning the practice of sterilization and disinfection of instruments used for patients according to clinical practice

As a result of comparing the degree of practice of sterilization and disinfection of instruments used for patients according to clinical practice sites, the 'ultrasonic scaler tip' scored the highest at 3.85 points, and the 'impression tray' was also the highest at 3.80 points. However, there was no statistically significant difference. University hospitals and general hospitals had the highest score of 4.00 for 'water instruments', 3.78 points for 'glass ball' and 3.91 points for 'rubber cup', which was higher in university hospitals and general hospitals than dental clinics and dental hospitals. There was no significant difference. "Cotton roll" dental hospitals scored 3.88 points and university hospitals and general hospitals scored 3.87 points, which was higher than that of dental clinics, with a statistically significant difference $(\mathrm{p}<0.00)$. 'Gauze' was higher at 3.96 points for university hospitals and general hospitals, showing a statistically significant difference than dental clinics $(\mathrm{p}<0.00)$ (Table 5).

\section{Discussion}

The environment of the dental office is always exposed to infectious factors, and cross-infection occurs between the dental worker and the patient through various routes such as direct contact with contaminated instruments and equipment, contact with aerosol, and direct contact with blood and saliva. I can. Therefore, dental hygienists must take responsibility and responsibility for infection prevention as medical personnel providing oral health services, and such infection prevention education must be conducted in schools before becoming dental hygienists [19]. Education through clinical practice can be defined as a process in which dental hygiene students learn to be recognized and recognized as future dental hygienists. In order to successfully prevent infection, students of dental hygiene, including dental health workers, have a correct understanding of infectious diseases, In other words, it is important to know the cause of the disease, infection route, vaccination, thorough sterilization method, practice effective alternative measures in case of injury, and willing to actively participate [20].

Therefore, this study aims to minimize infection in hospitals and to develop pre-clinical curriculum and education programs by investigating the perception and practice of dental infection prevention in actual clinical practice for dental hygiene students who have experienced clinical practice. It was implemented to obtain basic data for guidelines and practice guidelines. It can be seen that the number of experienced den- tal infection control education was high at $93.9 \%$, and the path of dental infection control education was most practically performed at $87.8 \%$ at dental practice sites. Therefore, it is thought that infection control education should be conducted in advance in schools. In addition, since the higher the experience in dental infection control education, the higher the degree of practice. Therefore, it is necessary to revitalize educational opportunities such as systematic institutional policy and maintenance education for infection control that can be actively practiced as well as correct recognition in infection control [21].

As for whether they had regular health check-ups before going to clinical practice, 59 people $(60.2 \%)$ answered 'yes', and 39 people (39.8\%) answered 'No'. The most common reason for not receiving regular health checkups was "because they thought they were healthy" with $80(81.6 \%)$, and 16 $(16.3 \%)$ were "because they are unlikely to be infected". As a result of this, it is thought that the motivation for dental infection management through school education is somewhat low. Therefore, it seems that it is necessary to provide thorough education for regular checkups before clinical practice. It seems that there is a high opinion that by following the infection prevention guidelines, you can thoroughly protect yourself from disease.

The most infectious diseases in dentistry were "HIV" 38 (38.8\%), "influenza" 31 (31.6\%), and "tuberculosis" 18 (18.4\%). The infection route of the clinical practice site was 'contaminated needles or sharp instruments' 42 (42.9\%), and 'through skin contact through blood, saliva and mucus' 34 (34.7\%). It is believed that the cause and route of infection should be more understood and the possibility of infection should be reduced through thorough education. It is thought that it is necessary to distribute infection prevention guidelines for trainees to institutions that practice training so that training staff manage the students in practice.

As a result of comparing matters on infection prevention attitudes according to clinical practice sites, university hospitals and general hospitals scored 3.96 points for unit chairs, 3.87 points for batting tables, and 3.91 points for protective equipment stained with saliva or blood. Dental clinics and dental hospitals showed high results.

In extract management, dental hospitals scored 3.88 points, and dental hospitals, university hospitals, and general hospitals were higher than dental clinics before and after treatment. In terms of hand washing, university hospitals and general hospitals scored 3.74 points for "before treatment" and 3.83 points for "after treatment", which was higher than that of dentists and dental hospitals.

Medical waste is a waste generated by health and medical 
institutions and requires special management, and has a task to focus on proper treatment rather than reuse through circulation [22]. There are three types of dental infectious waste, which must be stored and disposed of by dividing into damaging waste (injection needle, suture needle, surgical scalpel), cotton wool (gauze contaminated with body fluids such as blood, cotton wool, etc.) In the case of dental clinics, it was found to be lower, and institutional policies are needed to promote awareness education and practice on waste management [10].

University hospitals and general hospitals were higher than dental clinics and dental hospitals in both 'preparing for setting', 'before and after treatment' in surface disinfection. In both 'Towels', university hospitals and general hospitals were higher than dental clinics and dental hospitals. This is a situation in which the trainees are not provided with sufficient guidance and education when using protective equipment at the training institution, and as a trainee, there was a point that the implementation was not achieved due to various difficulties.

Hospital workers reported that there were cases where they could not follow the infection control guidelines due to 'lack of safety equipment and facilities related to work' and 'lack of equipment'. Therefore, if the training institution does not provide sufficient protective equipment, it is difficult for students to carry out the infection control guidelines. Therefore, financial support such as supply of goods or the basis for requesting the training institution should be prepared.

As a result of comparing the degree of practice of sterilization and disinfection of instruments used for patients according to clinical practice sites, it was found that "cotton roll" dental hospitals had 3.88 points and university hospitals and general hospitals had 3.87 points, which was higher than that of dental clinics. Therefore, infection control for instruments was well performed in university hospitals and general hospitals that treat more high-risk patients than dental clinics or dental hospitals. Most of the instruments used for patients in dentistry are sterilized in principle, and some instruments are used only by sterilization. In Korea's dentistry, infection control evaluation is not legally mandated, and due to the lack of an institutional system, it is subject to constant controversy [23]. Therefore, through the certification management system for each medical institution, it is an important measure in terms of safety issues for patients and medical workers, quality management of medical services, and infection control in hospitals, and the hospital certification system should also be expanded and implemented in dental clinics for the competitive advantage of hospitals. think.

The limitation of this study is that it is difficult to generalize it because it was investigated and studied with three-year universities in some regions. Therefore, it is thought that a more meaningful result would come out if a comparative study was conducted on the degree of performance through a thorough survey in the clinical practice sites of students in dental hygiene departments nationwide by expanding the region. In general, clinical practice is set as a regular subject in school, and practice is performed for actual patients in school practice, so vaccination before practice is mandatory for all students, and theory and clinical may be closely related. In particular, it is considered that a systematic infection control education program for students in the dental hygiene department is very necessary as a critical stepping bridge that can have a close effect on actual clinical work after graduation.

\section{Conclusion}

This study aims to reduce infections that can occur in dental clinics and identify problems in carrying out infection prevention guidelines during clinical practice, suggest improvement directions, and use them as basic data for education. The results of a questionnaire survey on the degree of recognition and performance of dental infection prevention are as follows.

$1.92 \%$ of clinical trainees received dental infection control education, and most of them were infection control education at clinical practice sites. $69.2 \%$ said 'yes' to whether they receive regular health checkups.

2. The most infectious disease among clinical trainees in dentistry was $\mathrm{HBV}$ at $38.8 \%$, and the reason for infection was "contaminated needles or sharp instruments" at $42.9 \%$.

3 . As a result of comparing the attitudes of infection prevention management according to clinical practice sites, university hospitals and general hospitals showed 3.96 points for unit chairs, 3.87 points for batting tables, and 3.91 points for protective equipment stained with saliva or blood. As points, there was a statistically significant difference between the dental clinic and the dental clinic $(\mathrm{p}<0.03)$. In extract management, dental hospitals scored 3.88 points, and dental hospitals, university hospitals, and general hospitals were higher than dental clinics before and after treatment. In terms of handwashing, university hospitals and general hospitals scored "before treatment" 3.74 points and "after treatment" 3.83 points, which were higher than dental clinics and dental hospitals. University hospitals and general hospitals were higher than dental clinics and dental hospitals in both 'preparing for setting', 'before and after treatment' in surface disinfection. In both 'towel', university hospitals and general hospitals were higher than dental clinics and dental hospitals.

4. As a result of comparing the degree of practice of steri- 
lization and disinfection of instruments used for patients according to clinical practice, the 'ultrasonic scaler tip' scored the highest at 3.85 points, and the 'impression tray' also scored 3.80 points. It was found to be the highest, but there was no statistically significant difference. University hospitals and general hospitals had the highest score of 4.00 for 'water instruments', 3.78 points for 'glass ball' and 3.91 points for 'rubber cup', which was higher in university hospitals and general hospitals than dental clinics and dental hospitals. There was no significant difference. "Cotton roll" dental hospitals scored 3.88 points and university hospitals and general hospitals scored 3.87 points, which was higher than that of dental clinics, with a statistically significant difference $(\mathrm{p}<$ 0.00). "Gauze" showed a high score of 3.96 for university hospitals and general hospitals, showing a statistically significant difference compared to dental clinics $(\mathrm{p}<0.00)$.

As a result of this study, in order to raise awareness and practice of infection control during clinical practice for dental hygiene students, guidelines for infection control were prepared and distributed so that training institutions can implement it during practice, and communicate well with the person in charge during the training period. To be able to do so, inspection and evaluation through continuous management should be carried out together. It is necessary to develop an infection control prevention program that is connected with schools and clinical practice institutions, and it is considered that the awareness and practice of infection control as well as the safety of dental hygienists must be improved through institutional improvement in infection control.

\section{Conflict of Interest}

No potential conflict of interest relevant to this article was reported.

\section{ORCID}

Min-Seo Kim, https://orcid.org/0000-0003-4206-3731

\section{References}

1. H. S Moon. A study on the health status of dentists. Journal of Korean Academy of Oral Health 1992;16:53-73.

2. Kwon- Suk Ahn, "Compliance with standard precaution among dental health care workers." Master, Chungnam University, 2005.

3. Yoon-Jung Cho, Seok-Jun Yoon, Hyeong-Sik Ahn, Soon-Duck Kim, Hyeung-Keun Park, "A Study on Infection Control Practice among Dental Hygienists", Journal of Korea Society of
Quality Assurance in Health Care 2003;10:190-204.

4. Y.S Nam and M, S Park. A study on practice Level of infection prevention of dental hygienist in dental clinics Journal of Korean Academy of Oral Health.

5. Jeong-Hee Park, Nam-suk Heo, Hye-Jeong Song, "A study of current infection control by dental hygienists and related factors." Journal of Korean society of Dental Hygiene 2011;11: 993-1003.

6. Ministry of health and welfare, "The criteria for infection prevention in dental clinic." 2006.

7. J.H Park, N. S Heo and H. J Song. A study of current infection control by dental hygienists and related factors. Journal of Korea society of Dental Hygiene 2011;11:993-1003.

8. J. K Kang, E. S Kim and K. M Kim. Study on the Infection Control and Dental Waste Disposal in Dental Clinic Located in Seoul City. Journal of Dental Hygiene Science 2002);2:105-13.

9. Y. S Cho, B. H Jun and Y. S Choi. Construction and Validation of Infection Control Practice Scale for Dental Hygienist. Journal of Dental Hygiene Science 2009;9:993-1003.

10. Young-Nam Park, Recognition and practice on infection control and infection waste of dental personnels, Master, Chungnam University, 2006.

11. Jung-Hyun Park, Kyeung-Ae Jang, "The Knowledge of infection control, practice and performance of dental hygienist." Journal of Korean society of Dental Hygiene 2012;12:953-61.

12. su-Jin Kwon, "Development of Evaluation Indicator for Dental Hospital Accreditation.” PhD, Gosin University, 2014.

13. Sang-Mi Nam, "A Study on perceived Level for pandemic Influenza and on Infection Control Practices by Dental Hygienists." Journal of Korean society of Dental Hygiene 2011; 11:135-46.

14. Mi-Suk Yoon, Mi-Suk Choi, "The Analysis of the Prevention against Virus Infection in Dental Hygienist at Medical Treatment." Journal of Korean Society of Dental Hygiene Science 2007;7:101-6.

15. Deok-Ja Lee, Sung-Hee Ko, Young-Hee Lee, "Perception and practice of hospital infection control in nurse of geriatric hospital: for convergent approach", Journal of digital convergence 2015;13:461-70.

16. Hyeon-Jeong Ju, “The effect of Nursing Students' Clinical practice Stress, Performance Ability, Satisfaction, and Critical thinking on Nursing prefessional Self concept.”, Journal of digital convergence 2017;15:213-24.

17. Lee SH. Infection control and hospital planing. The korean Soci Infec Dise 1990;22:199-206.

18. Young-Nam Park, "The study of awareness and performance of Dental hygiene students for infection control during clinical training." Journal of Digital Convergence 2017;15:307-15.

19. seon-jeong Moon, In-Young Ku, "Research on Cognition of Infection Control by Dental Hygienics Student in some Areas", 2012;12:313-320.

20. Maeng-soon Yu, "A study on the knowledge, attitudes, and preventive actions of dental hygiene college students on hospital infection.”, Master, KyungHee University, 2002.

21. Eun-sun Jung, “A study on dental Hygiene major's compliance 
with the infection control Guideeline during clinical practic.", Master, KyungHee University, 2013

22. Sae-Hee Ahn, Sang-Yoon Ahn, "The study of Area-division stategy for Medical Waste disposal." Journal of digital convergence 2014;12:255-63.
23. Sung-Suk Bae, Myung-Sun Lee, "Development of Evaluation Index for Infection Control and Prevention at Dental Hospital and Its Vlidity Verification" Journal of Korean society of Dental Hygiene 2013;13:254-63. 